\title{
The Mediterranean diet and fetal size parameters: the Generation R Study
}

\author{
Sarah Timmermans ${ }^{1,2 *}$, Régine P. Steegers-Theunissen ${ }^{2,3,4,5}$, Marijana Vujkovic ${ }^{2}$, \\ Hanneke den Breeijen ${ }^{3}$, Henk Russcher ${ }^{6}$, Jan Lindemans ${ }^{6}$, Johan Mackenbach ${ }^{7}$, Albert Hofman ${ }^{3}$, \\ Emmanuel E. Lesaffre ${ }^{8,9}$, Vincent V. Jaddoe ${ }^{1,3,4}$ and Eric A. Steegers ${ }^{2}$ \\ ${ }^{1}$ Generation R Study Group, Erasmus Medical Center, PO Box 2040, 3000CA Rotterdam, The Netherlands \\ ${ }^{2}$ Department of Obstetrics and Gynecology, Erasmus Medical Center, PO Box 2040, 3000CA Rotterdam, The Netherlands \\ ${ }^{3}$ Department of Epidemiology, Erasmus Medical Center, PO Box 2040, 3000CA Rotterdam, The Netherlands \\ ${ }^{4}$ Department of Pediatrics, Erasmus Medical Center, PO Box 2040, 3000CA Rotterdam, The Netherlands \\ ${ }^{5}$ Department of Clinical Genetics, Erasmus Medical Center, PO Box 2040, 3000CA Rotterdam, The Netherlands \\ ${ }^{6}$ Department of Clinical Chemistry, Erasmus Medical Center, PO Box 2040, 3000CA Rotterdam, The Netherlands \\ ${ }^{7}$ Department of Public Health, Erasmus Medical Center, PO Box 2040, 3000CA Rotterdam, The Netherlands \\ ${ }^{8}$ Department of Biostatistics, Erasmus Medical Center, PO Box 2040, 3000CA Rotterdam, The Netherlands \\ ${ }^{9}$ Biostatistical Centre, Catholic University Leuven, PO Box 5005, Leuven, Belgium
}

(Submitted 22 November 2011 - Final revision received 23 November 2011 - Accepted 23 November 2011 - First published online 21 February 2012)

\begin{abstract}
Developmental adaptations due to early nutritional exposures may have permanent health consequences. Studies of diet and fetal size have mainly focused on individual nutrients despite evidence that the pattern of food consumption may be of significance. Hence, we evaluated the associations of dietary habits in early pregnancy (gestational age $<18$ weeks) with fetal size, uteroplacental vascular resistance, placental weight and birth weight in a prospective observational study of 3207 Caucasian pregnant mothers in Rotterdam, the Netherlands. Participants completed a semiquantitative FFQ during early pregnancy. Logistic regression analysis was used to predict the occurrence of intra-uterine growth retardation at birth as a function of food intake. The derived solution was considered as the dietary pattern. As it was characterised by higher intakes of fruit, vegetables, vegetable oil, fish, pasta and rice, and lower intakes of meat, potatoes and fatty sauces, it was labelled the 'Mediterranean' diet. The degree of adherence to the diet was positively associated with plasma folate and serum vitamin $\mathrm{B}_{12}$ concentrations and showed an inverse relationship with homocysteine and high-sensitivity $\mathrm{C}$-reactive protein plasma concentrations $(P<0.05)$. Important fetal size and placental parameters were associated with the degree of adherence to the diet, revealing a $72 \mathrm{~g}$ lower birth weight $(95 \% \mathrm{CI}-110 \cdot 8,-33 \cdot 3)$ and a $15 \mathrm{~g}$ lower placental weight $(95 \% \mathrm{CI}-29 \cdot 8,-0 \cdot 2)$ for women with low adherence to the diet. To conclude, low adherence to a Mediterranean diet in early pregnancy seems associated with decreased intra-uterine size with a lower placental and a lower birth weight.
\end{abstract}

Key words: Dietary patterns: Birth weight: Folate: Placenta

Fetal growth is an important determinant of future health and development. Increasing evidence suggests that environmental exposures, acting at different stages of fetal development, can cause permanent developmental adaptations that may affect the physiology of various organ systems, leading to fetal growth retardation and increased risk of chronic diseases in later life $\mathrm{e}^{(1,2)}$. Maternal nutrition has been recognised as one of the most important extraneous stimuli influencing fetal growth and development.

During the past years, the identification of dietary patterns through data-driven methods has increasingly gained interest. The first studies have shown significant associations between dietary patterns and pregnancy complications ${ }^{(3-5)}$. In this respect, dietary patterns have also been related to biomarker concentrations in blood that are known to be important intermediates for placental and fetal growth and development, including folate and high-sensitivity C-reactive protein (Hs-CRP) $^{(3,6)}$.

Except for one study on birth weight, used as proxy for fetal growth, we are unaware of studies investigating the relationship between dietary intake in early pregnancy and growth adaptations during fetal life ${ }^{(7)}$. Therefore, we aimed to examine the associations of maternal diet with uteroplacental vascular resistance, placental weight, fetal size and birth weight.

Abbreviations: Hs-CRP, high-sensitivity C-reactive protein; IUGR, intra-uterine growth restriction; SDS, standard deviation score; tHcy, homocysteine.

*Corresponding author: Dr S. Timmermans, fax +31 107036815 , email s.timmermans@erasmusmc.nl 


\section{Methods}

Subjects

The present study was embedded in the Generation R Study, a population-based cohort study, from early pregnancy onwards ${ }^{(8,9)}$. Between 2001 and 2006, pregnant women living in Rotterdam, the Netherlands were invited to take part in the study. The present analysis was restricted to prenatally enrolled Dutch women with a spontaneously conceived live-born singleton pregnancy ( $n$ 3207; Fig. 1). The study was conducted following the World Medical Association Declaration of Helsinki ${ }^{(10)}$. Approval was obtained from the Medical Ethics Committee of the Erasmus Medical Center Rotterdam, the Netherlands. Every participant provided written informed consent.

\section{Dietary assessment}

Nutritional intake was assessed at enrolment (median 13.5 (interquartile range 3.4) weeks) using a modified version of the validated semiquantitative FFQ of Klipstein-Grobusch et $a l .{ }^{(11)}$. This FFQ covers food intake over the preceding 3 months. The FFQ consists of 293 items structured to meal pattern. Questions included consumption frequency, portion size preparation method and additions. Portion sizes were estimated using household measures and photographs ${ }^{(12)}$. To calculate the average daily nutritional values, the Dutch Food Composition Table 2006 was used ${ }^{(13)}$.

\section{Fetal and placental parameters}

Fetal ultrasound measurements plus medical records were used to obtain information about the outcome variables: fetal size until birth, placental weight, placental resistance and birth weight. Ultrasound measurements were used to establish gestational age in early pregnancy (gestational age $<18$ weeks) and to assess fetal biometry, including head circumference, abdominal circumference, femur length and estimated fetal weight in mid-pregnancy (gestational age

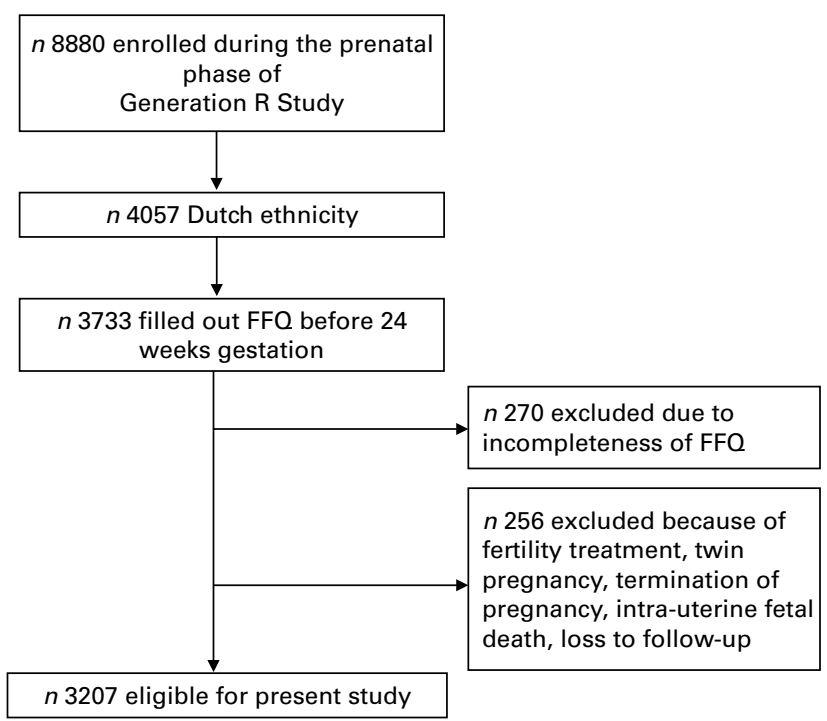

Fig. 1. Flow chart of the study population.
18-25 weeks) and late pregnancy (gestational age $\geq 25$ weeks). As described previously, these ultrasound measurements were used to construct growth charts and gestational age-adjusted standard deviation scores (SDS) ${ }^{(14)}$. The intraand inter-observer reproducibility scores of the ultrasound measurements have also been described before ${ }^{(15)}$. Using these growth charts, we defined intra-uterine growth restriction (IUGR) as a gestational age-adjusted birth weight below percentile-2.3 in the study cohort (SDS $<-2 \cdot 0$ ). Finally, uteroplacental resistance, measured by colour Doppler, was assessed by the Umbilical artery pulsatility index and the Uterine artery resistance index in late pregnancy.

\section{Biochemical analysis}

In early pregnancy, venous blood serum and plasma samples were drawn and stored at $-80^{\circ} \mathrm{C}^{(8)}$. Plasma folate, homocysteine (tHcy) and Hs-CRP, and serum vitamin $\mathrm{B}_{12}$ concentrations were analysed using a microparticle-enhanced immunoassay on the Architect system (Abbott Diagnostics B.V.) at the Department of Clinical Chemistry of the Erasmus Medical Center Rotterdam in 2008. The between-run CV were $8.9 \%$ at $5.6 \mathrm{nmol} / 1,2.5 \%$ at $16.6 \mathrm{nmol} / 1$ and $1.5 \%$ at $33.6 \mathrm{nmol} / 1$ (folate); $3.1 \%$ at $7.6 \mu \mathrm{mol} / 1,3.1 \%$ at $13.7 \mu \mathrm{mol} / 1$ and $2.1 \%$ at $26 \cdot 1 \mu \mathrm{mol} / 1$ (tHcy); $0.9 \%$ at $12.8 \mathrm{mg} / \mathrm{l}$ and $1.3 \%$ at $39.3 \mathrm{mg} / \mathrm{l}$ (Hs-CRP); and lastly, $3.6 \%$ at $148 \mathrm{pmol} / 1,2.7 \%$ at $295 \mathrm{pmol} / 1$ and $3 \cdot 1 \%$ at $590 \mathrm{pmol} / \mathrm{l}$ (vitamin $\mathrm{B}_{12}$ ). Biomarker concentrations were available in $78 \%$ of the study population ( $n$ 2519). No differences in nutritional intake were observed between women with and without biomarker concentrations $(P=0.76)$.

\section{Assessment of other variables}

Data on maternal age, education, parity, periconception folic acid use, vomiting during the first trimester and maternal comorbidity (defined as the occurrence of chronic hypertension/heart disease/diabetes/high cholesterol/thyroid disease/ systemic lupus erythematosus) were available from a questionnaire administered in the first trimester. Education was assessed by the highest completed education of the mother and classified as (1) low education; (2) middle education; (3) high education ${ }^{(16)}$. Information on smoking was obtained by self-administered questionnaires sent in the first, second and third trimesters. Smoking at enrolment was assessed in the first questionnaire by asking each mother whether she smoked during pregnancy (no smoking; first trimester only smoking; still smoking). This questionnaire was sent to all mothers, regardless of the gestational age at enrolment. To assess smoking habits in the second and third trimesters, the mothers were asked whether they smoked during the past 2 months (yes/no), in the second and third questionnaires. Mothers who reported in the first questionnaire that they smoked in the first trimester only, but still reported smoking in the second or third questionnaire, were reclassified into the 'continued smoking' category. The same strategy was used for mothers who reported no smoking in the first questionnaire, but reported smoking in the second or third 
Table 1. Associations between food groups and the degree of adherence to the Mediterranean diet (Correlation coefficients, medians and interquartile ranges)

\begin{tabular}{|c|c|c|c|c|c|c|c|c|c|}
\hline \multirow[b]{3}{*}{ Food group } & \multicolumn{9}{|c|}{ Adherence to the Mediterranean diet } \\
\hline & \multicolumn{3}{|c|}{ Total ( $n$ 3207) } & \multicolumn{2}{|c|}{ Low adherence $(n 1070)$} & \multicolumn{2}{|c|}{ Medium adherence ( $n$ 1069) } & \multicolumn{2}{|c|}{ High adherence ( $n$ 1068) } \\
\hline & $r$ & Median* $^{\star}$ & Interquartile range & Median* $^{*}$ & Interquartile range & Median* $^{*}$ & Interquartile range & Median* $^{*}$ & Interquartile range \\
\hline Pasta, rice & 0.61 & $46 \cdot 1$ & $27 \cdot 0-70 \cdot 5$ & $28 \cdot 5$ & $15 \cdot 5-44 \cdot 8$ & 44.0 & $29 \cdot 1-60 \cdot 4$ & $74 \cdot 2$ & $51 \cdot 6-99.7$ \\
\hline Bread & 0.33 & $133 \cdot 6$ & $101 \cdot 1-166 \cdot 9$ & 112.5 & $83 \cdot 3-144 \cdot 9$ & $137 \cdot 4$ & $104.9-167.8$ & $151 \cdot 3$ & $117 \cdot 4-181 \cdot 4$ \\
\hline Vegetable oil & 0.29 & $6 \cdot 1$ & $3 \cdot 2-9 \cdot 7$ & 4.4 & $2 \cdot 0-7 \cdot 8$ & 5.9 & $3 \cdot 3-9 \cdot 2$ & $7 \cdot 7$ & $4.8-11.5$ \\
\hline Fish & 0.26 & $10 \cdot 9$ & $5 \cdot 5-19 \cdot 0$ & 8.9 & $1 \cdot 6-15 \cdot 7$ & $10 \cdot 6$ & $4 \cdot 9-19 \cdot 1$ & $15 \cdot 8$ & $8 \cdot 8-21 \cdot 6$ \\
\hline Alcoholic drinks & 0.23 & 0.9 & $0-8.5$ & 0.6 & $0-6 \cdot 1$ & $1 \cdot 0$ & $0-8.0$ & $6 \cdot 0$ & $0 \cdot 1-21 \cdot 6$ \\
\hline Vegetables & 0.22 & $147 \cdot 1$ & $108 \cdot 9-189 \cdot 2$ & $131 \cdot 8$ & $96 \cdot 4-172 \cdot 7$ & $142 \cdot 1$ & $108 \cdot 9-182 \cdot 7$ & $164 \cdot 4$ & $128 \cdot 1-203 \cdot 2$ \\
\hline Fruit & 0.18 & $186 \cdot 7$ & $120 \cdot 9-242 \cdot 4$ & $167 \cdot 1$ & $99 \cdot 7-219 \cdot 3$ & $190 \cdot 5$ & $126 \cdot 1-246 \cdot 0$ & $200 \cdot 0$ & $139 \cdot 9-272 \cdot 0$ \\
\hline Breakfast cereals & 0.09 & $5 \cdot 2$ & $1.2-19 \cdot 6$ & 4.0 & $0.4-15 \cdot 0$ & $5 \cdot 7$ & $1 \cdot 6-21 \cdot 2$ & $5 \cdot 8$ & $1.5-20.9$ \\
\hline Soya and diet products & 0.07 & 0.1 & $0-0.4$ & 0.1 & $0-0.3$ & 0.1 & $0-0.4$ & 0.1 & $0-0.7$ \\
\hline Margarine & 0.06 & $18 \cdot 3$ & $7 \cdot 5-28 \cdot 7$ & $16 \cdot 6$ & $6 \cdot 8-26 \cdot 8$ & $18 \cdot 4$ & $8 \cdot 2-29 \cdot 3$ & $19 \cdot 6$ & $7 \cdot 0-30 \cdot 1$ \\
\hline Sweets & 0.0 & $97 \cdot 2$ & $71 \cdot 2-128.9$ & $97 \cdot 8$ & $69 \cdot 8-129 \cdot 3$ & $98 \cdot 1$ & $72 \cdot 3-131 \cdot 2$ & $96 \cdot 1$ & $70 \cdot 7-127 \cdot 2$ \\
\hline Butter & -0.02 & 0.4 & $0-1 \cdot 3$ & 0.4 & $0-1.4$ & 0.4 & $0-1.3$ & 0.3 & $0-1 \cdot 1$ \\
\hline Dairy products & -0.03 & 455.5 & $315 \cdot 4-601 \cdot 2$ & $465 \cdot 0$ & $308 \cdot 4-616 \cdot 4$ & $462 \cdot 3$ & $319 \cdot 4-602 \cdot 7$ & 441.9 & $316.6-586.9$ \\
\hline Non-alcoholic drinks & -0.03 & 1359.5 & $1002 \cdot 4-1780 \cdot 0$ & 1394.4 & $1002 \cdot 4-1881 \cdot 6$ & 1343.5 & $994 \cdot 8-1734 \cdot 6$ & $1342 \cdot 8$ & $1009 \cdot 9-1733 \cdot 8$ \\
\hline Soup & -0.03 & $45 \cdot 7$ & $20 \cdot 5-94.9$ & $48 \cdot 0$ & $21 \cdot 3-103 \cdot 1$ & $44 \cdot 8$ & $21 \cdot 4-90 \cdot 1$ & 44.4 & $19 \cdot 2-91 \cdot 7$ \\
\hline Starches and wheat & -0.04 & 0.7 & $0-8.6$ & 0.9 & $0-8 \cdot 6$ & 0.7 & $0-8 \cdot 8$ & 0.6 & $0-8.6$ \\
\hline Legumes & -0.09 & $1 \cdot 1$ & $0.1-7.5$ & 2.5 & $0.2-8 \cdot 1$ & 1.2 & $0.1-7.3$ & 0.6 & $0-7.3$ \\
\hline Eggs & -0.11 & $9 \cdot 7$ & $6 \cdot 2-14 \cdot 4$ & $10 \cdot 9$ & $6 \cdot 6-15 \cdot 8$ & 9.5 & $6 \cdot 3-13 \cdot 8$ & 9.1 & $5 \cdot 8-13.5$ \\
\hline Potatoes & -0.26 & $51 \cdot 6$ & $29 \cdot 1-79 \cdot 1$ & $65 \cdot 7$ & $38 \cdot 1-96 \cdot 1$ & $50 \cdot 2$ & $29 \cdot 9-78 \cdot 3$ & $41 \cdot 6$ & $22 \cdot 9-64 \cdot 2$ \\
\hline Meat & -0.30 & $84 \cdot 8$ & $59 \cdot 5-110 \cdot 3$ & $98 \cdot 4$ & $74 \cdot 2-123 \cdot 6$ & 83.5 & $62 \cdot 0-105 \cdot 6$ & 72.5 & $47 \cdot 8-98 \cdot 3$ \\
\hline Sauces and condiments & -0.36 & $26 \cdot 7$ & $18 \cdot 0-37 \cdot 2$ & $33 \cdot 8$ & $23 \cdot 7-46 \cdot 5$ & $25 \cdot 9$ & $17 \cdot 8-35 \cdot 9$ & 21.8 & $14 \cdot 8-30 \cdot 0$ \\
\hline
\end{tabular}

$r$, Spearman's rank correlation coefficient.

${ }^{*}$ Median daily intake in $\mathrm{g} / \mathrm{d}$ adjusted for energy intake. 
questionnaire $^{(17)}$. At enrolment, maternal height and weight were measured to calculate BMI $\left(\mathrm{kg} / \mathrm{m}^{2}\right)$. Information on fertility treatment, sex of infant and pregnancy outcome was obtained from midwives and obstetricians.

\section{Statistical analysis}

Logistic regression analysis was used to predict the occurrence of IUGR (yes/no (0/1)) as a function of food intake. To arrive at the logistic regression solution, the 293 food items were first reduced to twenty-one predefined food groups ${ }^{(3)}$. Subsequently, since food consumption is usually highly correlated, we calculated energy-adjusted food group intakes using the regression residual $\operatorname{method}^{(18)}$. Then, logistic regression analysis was performed to identify the dietary pattern predicting IUGR. In this study, the probability for IUGR seemed to express adherence to a Mediterranean-like diet. Hence, here we call the logistic regression solution:

Table 2. Baseline characteristics*

(Mean values and standard deviations; medians and interquartile ranges; percentages)

\begin{tabular}{|c|c|c|c|c|}
\hline & \multicolumn{4}{|c|}{ Mediterranean diet } \\
\hline & Low adherence ( $n$ 1070) & Medium adherence ( $n$ 1068) & High adherence ( $n$ 1069) & $P$ \\
\hline Mean maternal age (years) & & & & $<0.001$ \\
\hline Mean & $30 \cdot 2$ & 31.5 & $32 \cdot 3$ & \\
\hline SD & 4.8 & $4 \cdot 2$ & 3.7 & \\
\hline BMI $\left(\mathrm{kg} / \mathrm{m}^{2}\right)$ & & & & $<0.001$ \\
\hline Median & 23.9 & $23 \cdot 3$ & $22 \cdot 8$ & \\
\hline Interquartile range & $21 \cdot 8-26 \cdot 9$ & $21 \cdot 6-25 \cdot 7$ & $21 \cdot 3-25 \cdot 0$ & \\
\hline \multicolumn{5}{|l|}{ Education (\%) } \\
\hline Low & 6.5 & $2 \cdot 0$ & 0.7 & $<0.001$ \\
\hline Medium & $55 \cdot 0$ & $34 \cdot 1$ & $22 \cdot 7$ & \\
\hline High & 37.9 & 63.5 & $76 \cdot 0$ & \\
\hline Missing & 0.6 & 0.5 & 0.6 & \\
\hline \multicolumn{5}{|l|}{ Parity (\%) } \\
\hline 0 & $60 \cdot 9$ & $60 \cdot 6$ & 58.9 & 0.280 \\
\hline$\geq 1$ & $39 \cdot 1$ & $39 \cdot 2$ & $41 \cdot 1$ & \\
\hline Missing & 0 & 0.2 & 0 & \\
\hline \multicolumn{5}{|l|}{ Smoking (\%) } \\
\hline Yes, still & $25 \cdot 1$ & $10 \cdot 6$ & $9 \cdot 4$ & $<0.001$ \\
\hline Yes, stopped & 8.2 & 8.7 & $8 \cdot 2$ & \\
\hline No & $60 \cdot 0$ & $73 \cdot 8$ & $75 \cdot 9$ & \\
\hline Missing & $6 \cdot 6$ & 6.9 & 8.2 & \\
\hline \multicolumn{5}{|l|}{ Folic acid use (\%) } \\
\hline No & $12 \cdot 7$ & $7 \cdot 7$ & $6 \cdot 8$ & $<0.001$ \\
\hline Yes, postconception start & $27 \cdot 0$ & 28.5 & 28.5 & \\
\hline Yes, preconception start & $43 \cdot 1$ & $46 \cdot 2$ & 49.5 & \\
\hline Missing & $17 \cdot 2$ & $17 \cdot 7$ & $15 \cdot 2$ & \\
\hline \multicolumn{5}{|l|}{ Vomiting (\%) } \\
\hline Severe & $16 \cdot 2$ & $13 \cdot 4$ & $9 \cdot 1$ & $<0.001$ \\
\hline Moderate & $20 \cdot 7$ & $19 \cdot 6$ & $20 \cdot 3$ & \\
\hline No & $55 \cdot 7$ & $59 \cdot 7$ & $63 \cdot 6$ & \\
\hline Missing & 7.4 & $7 \cdot 3$ & $7 \cdot 0$ & \\
\hline \multicolumn{5}{|l|}{ Comorbidity (\%) } \\
\hline Yes & $7 \cdot 0$ & 4.5 & 4.9 & $<0.025$ \\
\hline No & 94.0 & 88.9 & $88 \cdot 3$ & \\
\hline Missing & $6 \cdot 0$ & $6 \cdot 6$ & $6 \cdot 8$ & \\
\hline Male sex (\%) & 50.5 & 49.5 & $51 \cdot 7$ & 0.596 \\
\hline IUGR (\%) & 4.7 & 1.8 & 1.7 & $<0.001$ \\
\hline $\mathrm{PI}$ arteria umbilicalis & & & & 0.005 \\
\hline Mean & 0.99 & 0.98 & 0.96 & \\
\hline SD & 0.20 & 0.16 & 0.15 & \\
\hline $\mathrm{RI}$ arteria uterina & & & & 0.043 \\
\hline Mean & 0.49 & 0.47 & 0.48 & \\
\hline SD & 0.18 & 0.08 & 0.08 & \\
\hline Placental weight (g) & & & & 0.022 \\
\hline Mean & 629.5 & $648 \cdot 2$ & $646 \cdot 6$ & \\
\hline SD & $143 \cdot 8$ & $148 \cdot 9$ & $148 \cdot 3$ & \\
\hline Gestational age (weeks) & & & & 0.056 \\
\hline Median & $40 \cdot 1$ & $40 \cdot 3$ & $40 \cdot 3$ & \\
\hline Interquartile range & $39 \cdot 0-40 \cdot 9$ & $39 \cdot 3-41 \cdot 1$ & $39 \cdot 3-41 \cdot 1$ & \\
\hline Birth weight $(\mathrm{g})$ & & & & $<0.001$ \\
\hline Mean & $3424 \cdot 3$ & 3514.4 & $3521 \cdot 7$ & \\
\hline SD & $566 \cdot 1$ & $557 \cdot 9$ & $536 \cdot 7$ & \\
\hline
\end{tabular}

IUGR, intra-uterine growth restriction; PI, pulsatility index; RI, resistance index.

${ }^{*}$ ANOVA and $\chi^{2}$ test tested overall differences in baseline characteristics between the three dietary pattern categories. 
'adherence to the Mediterranean diet'. For reasons of interpretability, all women were categorised into equal tertiles based on their probability score for the diet, namely: (1) low adherence; (2) medium adherence; (3) high adherence. To test differences in baseline characteristics between the three diet adherence categories, the ANOVA and $\chi^{2}$ test were used.

We assumed that nutrition affects growth processes underlying IUGR in a comparable manner as fetal and placental growth. Therefore, linear regression was used to assess cross-sectional differences between the diet adherence categories in (1) fetal size characteristics and (2) placental parameters. In the multiple regression analyses, the inclusion of confounding variables was based on earlier literature, and determined a priori. These were maternal age, parity, education, height, weight, smoking, folic acid use, vomiting, comorbidity, sex and gestational age. Potential confounders were selected if the effect estimates changed $\geq 10 \%$ in exploratory analyses. By using this approach, vomiting and comorbidity were not included into the final multiple analyses. Missing data on the missing covariables BMI $(0.4 \%)$, educational level $(0.5 \%)$, parity $(0.1 \%)$, smoking $(6.7 \%)$, folic acid use (16.7\%), vomiting (7.2\%) and comorbidity (6.4\%) were completed using the Markov Chain Monte Carlo multiple imputation technique ${ }^{(19)}$. Overall, five completed data sets were created. Subsequently, multiple regression analyses were performed separately on each completed data set and thereafter combined to one pooled estimate $^{(20)}$.

Lastly, effect modification was tested by multiplying the personal diet scores with the covariables educational level, parity, smoking, BMI and periconception folic acid use. If $P<0 \cdot 10$ was fulfilled, then multiple linear regression analyses were performed in the strata of that specific determinant.

The statistical software package SPSS 17.0 (SPSS, Inc.) was used for data analyses.

\section{Results}

The identified Mediterranean diet was characterised by higher intakes of pasta, rice, vegetable oils, fish, vegetables and alcohol, and lower intakes of meat, potatoes and fatty sauces $(r \geq 0 \cdot 20$; Table 1$)$.

Maternal characteristics associated with low adherence to the diet were younger maternal age, higher BMI, lower educational level, a lower frequency of folic acid use, and continued smoking (Table 2).

Lower folate and vitamin $\mathrm{B}_{12}$ concentrations were observed among women with low adherence to the diet (Table 3). Low adherence was also associated with higher tHcy and Hs-CRP concentrations. Compared to women with high adherence, women with a low adherence had a higher percentage of energy derived from fat and a lower ratio of unsaturated-tosaturated lipids. These women also consumed relatively

Table 3. Biomarker concentrations and nutrient intakes

(Medians and interquartile ranges)

\begin{tabular}{|c|c|c|c|c|c|c|}
\hline & \multicolumn{6}{|c|}{ Mediterranean diet } \\
\hline & \multicolumn{2}{|c|}{ Low adherence } & \multicolumn{2}{|c|}{ Medium adherence } & \multicolumn{2}{|c|}{ High adherence } \\
\hline & Median & Interquartile range & Median & Interquartile range & Median & Interquartile range \\
\hline \multicolumn{7}{|l|}{ Biomarker concentrations } \\
\hline$n$ & & 847 & & 837 & & 836 \\
\hline Folate $(\mathrm{nmol} / \mathrm{l})$ & $18 \cdot 2$ & $11 \cdot 5-25 \cdot 4$ & $18 \cdot 8$ & $12 \cdot 7-25 \cdot 4$ & $20 \cdot 0$ & $13 \cdot 5-26 \cdot 8$ \\
\hline $\mathrm{tHcy}(\mu \mathrm{mol} / \mathrm{l})$ & $7 \cdot 1$ & $6 \cdot 2-8 \cdot 3$ & $7 \cdot 0$ & $6 \cdot 1-8 \cdot 0$ & $7 \cdot 0$ & $6 \cdot 1-7 \cdot 8$ \\
\hline Vitamin $\mathrm{B}_{12}(\mathrm{pmol} / \mathrm{l})$ & $168 \cdot 5$ & $128 \cdot 3-225 \cdot 0$ & 178.0 & $133 \cdot 0-234 \cdot 5$ & $180 \cdot 0$ & $141 \cdot 0-244 \cdot 3$ \\
\hline $\mathrm{Hs}-\mathrm{CRP}(\mathrm{mg} / \mathrm{l})$ & $4 \cdot 8$ & $2 \cdot 6-8 \cdot 4$ & $4 \cdot 1$ & $2.2-7.9$ & 3.6 & $2 \cdot 0-6 \cdot 3$ \\
\hline \multicolumn{7}{|l|}{ Energy and macronutrients } \\
\hline$n$ & & 1070 & & 1068 & & 1069 \\
\hline Energy (kJ/d) & $8969 \cdot 7$ & $7689 \cdot 0-10523 \cdot 2$ & $8817 \cdot 0$ & $7292 \cdot 0-10266 \cdot 8$ & $8968 \cdot 7$ & $7320 \cdot 6-10631 \cdot 9$ \\
\hline Fat (\% of energy) & $37 \cdot 0$ & $32 \cdot 2-39 \cdot 1$ & $36 \cdot 5$ & $32 \cdot 6-39 \cdot 9$ & $35 \cdot 8$ & $33 \cdot 1-40 \cdot 6$ \\
\hline Total fat $(\mathrm{g} / \mathrm{d})^{*}$ & $80 \cdot 2$ & $69 \cdot 7-84 \cdot 7$ & $79 \cdot 0$ & $70 \cdot 7-86 \cdot 5$ & $77 \cdot 6$ & $71 \cdot 6-88.0$ \\
\hline Saturated lipids $(g / d)^{*}$ & 30.0 & $24.9-31.3$ & 28.9 & $25 \cdot 7-32 \cdot 3$ & $28 \cdot 0$ & $26 \cdot 5-33 \cdot 4$ \\
\hline Monounsaturated lipids $(\mathrm{g} / \mathrm{d})^{\star}$ & $28 \cdot 7$ & $24 \cdot 7-31 \cdot 4$ & $28 \cdot 3$ & $25 \cdot 0-31 \cdot 7$ & $28 \cdot 0$ & $25 \cdot 6-32 \cdot 4$ \\
\hline Polyunsaturated lipids $(\mathrm{g} / \mathrm{d})^{*}$ & $17 \cdot 8$ & $14.8-21.5$ & $18 \cdot 3$ & $15 \cdot 1-21 \cdot 8$ & $18 \cdot 2$ & $14 \cdot 8-21 \cdot 3$ \\
\hline Linoleic acid $(g / d)^{\star}$ & $14 \cdot 2$ & $11.9-17.8$ & $14 \cdot 8$ & $12 \cdot 0-17 \cdot 9$ & $14 \cdot 7$ & $11 \cdot 5-17 \cdot 1$ \\
\hline Cholesterol $(\mathrm{mg} / \mathrm{d})^{\star}$ & $176 \cdot 1$ & $128 \cdot 6-181 \cdot 9$ & $160 \cdot 3$ & $134.6-189.4$ & $154 \cdot 7$ & $146 \cdot 9-209 \cdot 2$ \\
\hline Ratio unsaturated lipids:saturated lipids & 1.5 & $1.4-1.9$ & 1.6 & $1.4-1.9$ & 1.7 & $1.4-1.8$ \\
\hline Protein (\% of energy) & 14.5 & $13 \cdot 7-16 \cdot 5$ & 14.9 & $13 \cdot 4-16 \cdot 4$ & $15 \cdot 1$ & $12 \cdot 9-16 \cdot 1$ \\
\hline Total protein $(\mathrm{g} / \mathrm{d})^{*}$ & 71.8 & $68 \cdot 1-81 \cdot 2$ & 73.1 & $66.5-80.5$ & 74.6 & $63 \cdot 9-79 \cdot 2$ \\
\hline Vegetable protein $(\mathrm{g} / \mathrm{d})^{*}$ & $25 \cdot 1$ & $27 \cdot 4-33 \cdot 2$ & 28.5 & $25 \cdot 4-31 \cdot 2$ & $30 \cdot 1$ & $21 \cdot 9-28 \cdot 6$ \\
\hline Animal protein $(\mathrm{g} / \mathrm{d})^{*}$ & 45.5 & $36 \cdot 7-51 \cdot 3$ & 44.4 & $38 \cdot 0-52 \cdot 1$ & 44.4 & $39 \cdot 0-53 \cdot 4$ \\
\hline Carbohydrate ( $\%$ of energy) & $48 \cdot 2$ & $44.9-52 \cdot 4$ & $48 \cdot 3$ & $44 \cdot 4-52 \cdot 3$ & 48.4 & $43 \cdot 8-52 \cdot 4$ \\
\hline Total carbohydrate $(\mathrm{g} / \mathrm{d})^{*}$ & 233.9 & $218 \cdot 2-255 \cdot 2$ & $235 \cdot 0$ & $216 \cdot 1-254 \cdot 9$ & 234.5 & $213 \cdot 5-255 \cdot 6$ \\
\hline Carbohydrate monosaccharides $(\mathrm{g} / \mathrm{d})^{\star}$ & $135 \cdot 9$ & $103 \cdot 6-139 \cdot 7$ & $127 \cdot 5$ & $109 \cdot 3-148 \cdot 9$ & $121 \cdot 2$ & $114 \cdot 3-157 \cdot 9$ \\
\hline Carbohydrate polymers $(\mathrm{g} / \mathrm{d})^{\star}$ & $95 \cdot 7$ & $101 \cdot 6-123 \cdot 2$ & $104 \cdot 6$ & $94 \cdot 6-115 \cdot 8$ & $111 \cdot 7$ & $84.4-107.9$ \\
\hline Fibre $(g / d)^{\star}$ & $19 \cdot 2$ & $20 \cdot 5-26 \cdot 4$ & $21 \cdot 8$ & $18 \cdot 9-24 \cdot 6$ & $23 \cdot 3$ & $16 \cdot 3-22 \cdot 2$ \\
\hline
\end{tabular}

tHcy, homocysteine; Hs-CRP, high-sensitivity C-reactive protein.

* Adjusted for energy intake. 
Table 4. Associations between the degree of adherence to the Mediterranean diet and placental parameters (Regression coefficients and $95 \%$ confidence intervals)

\begin{tabular}{|c|c|c|c|c|c|c|c|c|c|c|c|c|}
\hline \multirow[b]{3}{*}{ Mediterranean diet } & \multicolumn{4}{|c|}{ Placental weight } & \multicolumn{4}{|c|}{ Pulsatility index umbilical artery } & \multicolumn{4}{|c|}{ Resistance index uterine artery } \\
\hline & \multicolumn{2}{|c|}{ Crude† } & \multicolumn{2}{|c|}{ Adjusted $\ddagger$} & \multicolumn{2}{|c|}{ Crude† } & \multicolumn{2}{|c|}{ Adjusted $\ddagger$} & \multicolumn{2}{|c|}{ Crude† } & \multicolumn{2}{|r|}{ Adjusted $\ddagger$} \\
\hline & $\beta$ & $95 \% \mathrm{Cl}$ & $\beta$ & $95 \% \mathrm{Cl}$ & $\beta$ & $95 \% \mathrm{Cl}$ & $\beta$ & $95 \% \mathrm{Cl}$ & $\beta$ & $95 \% \mathrm{Cl}$ & $\beta$ & $95 \% \mathrm{Cl}$ \\
\hline Low adherence & $-12 \cdot 2$ & $-26 \cdot 5,2 \cdot 1$ & $-15 \cdot 0$ & $-29.8,-0.2$ & 0.02 & $0.01,0.04$ & 0.01 & $-0.00,0.03$ & 0.01 & $0.00,0.03$ & 0.01 & $-0.00,0.03$ \\
\hline Medium adherence & $1 \cdot 1$ & $-13 \cdot 1,15 \cdot 2$ & -0.78 & $-14 \cdot 9,13 \cdot 3$ & 0.02 & $0.00,0.03$ & -0.01 & $0.01,-0.02$ & -0.00 & $-0.02,0.01$ & 0.02 & $-0.00,0.03$ \\
\hline High adherence & \multicolumn{2}{|c|}{ Reference } & \multicolumn{2}{|c|}{ Reference } & \multicolumn{2}{|c|}{ Reference } & \multicolumn{2}{|c|}{ Reference } & \multicolumn{2}{|c|}{ Reference } & \multicolumn{2}{|c|}{ Reference } \\
\hline Linear trend analyses & \multirow{2}{*}{\multicolumn{4}{|c|}{$\begin{array}{c}179 \cdot 2 \\
28 \cdot 7.329 \cdot 8\end{array}$}} & \multirow{2}{*}{\multicolumn{4}{|c|}{$\begin{array}{c}-0.28 \\
-0.46-0.10\end{array}$}} & \multirow{2}{*}{\multicolumn{4}{|c|}{$\begin{array}{c}-0.09 \\
-0.240 .06\end{array}$}} \\
\hline $\begin{array}{l}\beta \\
95 \% \mathrm{Cl}\end{array}$ & & & & & & & & & & & & \\
\hline
\end{tabular}

$\beta$, Regression coefficient.

* Results from linear regression analyses. All presented values reflect the difference in grams for placental weight, or the difference in resistance/pulsatility index in late pregnancy, compared to the reference category 'high adherence'. Linear trend analyses reflect the change in placental weight in grams, or the change in the resistance/pulsatility index per unit (factor score) change. Uteroplacental resistance/pulsatility index analyses are based on, respectively, 2613 Umbilical artery Doppler measurements and 1956 Uterine artery Doppler measurements.

or gestational age.

$\ddagger$ Additionally adjusted for maternal age, height, weight, parity, sex, education, smoking and folic acid use.

Table 5. Associations between the degree of adherence to the Mediterranean diet and fetal size characteristics* (Regression coefficients and $95 \%$ confidence intervals)

\begin{tabular}{|c|c|c|c|c|c|c|c|c|c|c|c|c|c|c|c|c|}
\hline \multirow[b]{3}{*}{ Mediterranean diet } & \multicolumn{4}{|c|}{ SDS head circumference } & \multicolumn{4}{|c|}{ SDS abdominal circumference } & \multicolumn{4}{|c|}{ SDS femur length } & \multicolumn{4}{|c|}{ SDS estimated fetal weight } \\
\hline & \multicolumn{2}{|r|}{ Crudet } & \multicolumn{2}{|c|}{ Adjusted $\neq$} & \multicolumn{2}{|r|}{ Crude† } & \multicolumn{2}{|r|}{ Adjusted $\neq$} & \multicolumn{2}{|r|}{ Crude } & \multicolumn{2}{|r|}{ Adjusted $\neq$} & \multicolumn{2}{|r|}{ Crude† } & \multicolumn{2}{|r|}{ Adjusted $\neq$} \\
\hline & $\beta$ & $95 \% \mathrm{Cl}$ & $\beta$ & $95 \% \mathrm{Cl}$ & $\beta$ & $95 \% \mathrm{Cl}$ & $\beta$ & $95 \% \mathrm{Cl}$ & $\beta$ & $95 \% \mathrm{Cl}$ & $\beta$ & $95 \% \mathrm{Cl}$ & $\beta$ & $95 \% \mathrm{Cl}$ & $\beta$ & $95 \% \mathrm{Cl}$ \\
\hline \multicolumn{17}{|l|}{ Mid-pregnancy } \\
\hline Low adherence & -0.02 & $-0.11,0.07$ & 0.01 & $-0.09,0.10$ & -0.08 & $-0.16,0.01$ & -0.05 & $-0.14,0.04$ & 0.11 & $0.02,0.19$ & 0.07 & $-0.02,0.16$ & -0.00 & $-0.09,0.09$ & -0.01 & $-0.09,-0.08$ \\
\hline $\begin{array}{l}\text { Medium adherence } \\
\text { High adherence }\end{array}$ & -0.00 & $\begin{array}{l}-0.09,0.09 \\
\text { Reference }\end{array}$ & 0.01 & $\begin{array}{l}-0.08,0.10 \\
\text { ieference }\end{array}$ & -0.04 & $\begin{array}{l}-0.13,0.05 \\
\text { Reference }\end{array}$ & -0.03 & $\begin{array}{l}-0.12,0.06 \\
\text { Reference }\end{array}$ & 0.05 & $\begin{array}{l}-0.04,0.13 \\
\text { Reference }\end{array}$ & 0.02 & $\begin{array}{l}-0.06,0.11 \\
\text { Reference }\end{array}$ & 0.01 & $\begin{array}{l}-0.08,0.09 \\
\text { Reference }\end{array}$ & 0.08 & $\begin{array}{l}-0.08,0.09 \\
\text { Reference }\end{array}$ \\
\hline $\begin{array}{l}\text { Linear trend analyses } \\
\quad \beta \\
95 \% \mathrm{Cl}\end{array}$ & \multirow{2}{*}{\multicolumn{4}{|c|}{$\begin{array}{c}0.73 \\
-0.19,1.66\end{array}$}} & \multirow{2}{*}{\multicolumn{4}{|c|}{$\begin{array}{c}1 \cdot 07 \\
0.18,2 \cdot 25\end{array}$}} & \multirow{2}{*}{\multicolumn{4}{|c|}{$\begin{array}{c}-1.07 \\
-1.96,-0.19\end{array}$}} & \multirow{2}{*}{\multicolumn{4}{|c|}{$\begin{array}{c}0.19 \\
-0.70,1 \cdot 10\end{array}$}} \\
\hline Late-pregnancy & & & & & & & & & & & & & & & & \\
\hline Low adherence & -0.11 & $-0.20,-0.02$ & -0.08 & $-0.17,0.01$ & -0.16 & $-0.24,-0.07$ & -0.16 & $-0.25,-0.07$ & 0.01 & $-0.08,0.09$ & 0.01 & $-0.08,0.09$ & -0.10 & $-0.19,-0.02$ & -0.11 & $-0.20,-0.02$ \\
\hline $\begin{array}{l}\text { Medium adherence } \\
\text { High adherence }\end{array}$ & -0.04 & $-0.12,0.05$ & -0.03 & $-0.12,0.05$ & -0.05 & $-0.14,0.03$ & -0.06 & $-0.15,0.03$ & 0.01 & $-0.07,0.10$ & 0.00 & $-0.08,0.08$ & -0.08 & $-0.17,0.01$ & -0.07 & $-0.16,0.02$ \\
\hline Linear trend analyses & \multirow{2}{*}{\multicolumn{4}{|c|}{$\begin{array}{c}1 \cdot 34 \\
0.43,2 \cdot 25\end{array}$}} & \multirow{2}{*}{\multicolumn{4}{|c|}{$\begin{array}{c}1.71 \\
0.80,2 \cdot 62\end{array}$}} & & reterence & & Reference & & Reference & & Reference \\
\hline$\beta$ & & & & & & & & & \multicolumn{4}{|c|}{$\begin{array}{c}0.21 \\
-0.66,1 \cdot 10\end{array}$} & \multicolumn{4}{|c|}{$\begin{array}{c}1 \cdot 25 \\
0.33,2 \cdot 17\end{array}$} \\
\hline
\end{tabular}

SDS, standard deviation score; $\beta$, regression coefficient.

${ }^{*}$ Results from linear regression analyses. All presented values reflect the difference in gestational-age-adjusted SDS of each growth charateristic, compared to reference category 'high adherence'. Linear trend analyses reflect the change in gestational-age-adjusted SDS of each growth charateristic per unit (factor score) change. Analyses are based on, respectively, 3138 head circumference meaurements, 3139 abdominal circumference measurements and 3136 femur length measurements in mid-pregnancy; and 3111 head circumference measurements, 3136 abdominal circumference measurements and 3145 femur length measurements in late pregnancy.

† Adjusted for gestational age.

$\ddagger$ Additionally adjusted for maternal age, height, weight, parity, sex, education, smoking and folic acid use. 


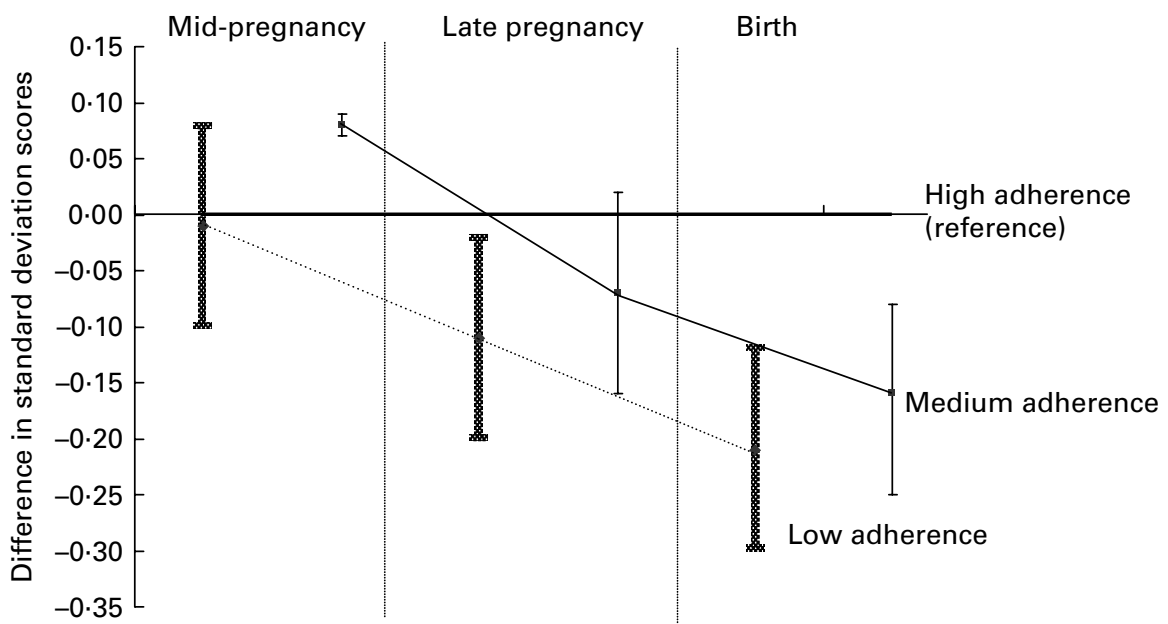

Fig. 2. Associations between the degree of adherence to the Mediterranean diet and estimated fetal weight and birth weight. Results from linear regression analyses. Values reflect the differences in gestational age-adjusted standard deviation scores of estimated fetal weight/birth weight in mid-pregnancy $(n 3133$ measurements), and late pregnancy ( $n 3121$ measurements), and at birth ( $n 3195$ measurements) for fetuses/infants of women with low or medium adherence to the Mediterranean diet, relative to fetuses of women with high adherence (reference). All presented values are adjusted for maternal age, height, weight, parity, sex, education, smoking, and folic acid use

lower amounts of vegetable protein, carbohydrate polymers and fibre.

Low adherence to the Mediterranean diet was associated with a $15 \mathrm{~g}$ lower placental weight compared to women with high adherence to the diet (95\% CI $-29 \cdot 8,-0 \cdot 2$; Table 4). A similar trend with respect to uteroplacental vascular resistance was observed (per unit (factor score) change: -0.28 pulsatility index change (95\% CI $-0 \cdot 46,-0 \cdot 10)$.

Low adherence to the Mediterranean diet was associated with smaller abdominal circumference in late pregnancy (difference in SDS $=-0 \cdot 16,95 \% \mathrm{CI}-0 \cdot 25,-0 \cdot 07$; Table 5). A similar, but not significantly, trend towards a smaller head circumference in late pregnancy was also observed for women with a low adherence (difference in SDS $=-0.08$, 95\% CI $-0 \cdot 17,0 \cdot 01)$.

Table 5 and Fig. 2 show the differences in the SDS of estimated fetal weight and birth weight between the three diet categories. Low adherence to the Mediterranean diet was associated with reduced fetal size from late-pregnancy onwards (difference in SDS at 30 weeks $=-0 \cdot 11,95 \% \mathrm{CI}$ $-0 \cdot 20,-0 \cdot 02)$. This difference became more pronounced at birth, revealing a $72 \mathrm{~g}$ lower birth weight in infants of women with low adherence to the Mediterranean diet (difference in grams at birth $=-72 \cdot 0,95 \% \mathrm{CI}-110 \cdot 8,-33 \cdot 3)$ and difference in SDS at birth $=-0 \cdot 21,95 \% \mathrm{CI}-0 \cdot 30,-0 \cdot 12$ ) Medium adherence was also significantly associated with reduced birth weight (difference in grams at birth $=-58.0$, $95 \%$ CI $-95.8,-20.3)$ and difference in SDS at birth $=-0 \cdot 16,95 \%$ CI $-0 \cdot 24,-0 \cdot 07)$. These values are adjusted for potential confounders.

Educational level and smoking modified the associations between the Mediterranean diet and birth weight (both interaction terms $P<0 \cdot 10$ ). Compared to high-educated women with high adherence, low adherence to the diet was associated with a $131 \mathrm{~g}$ lower birth weight (95\% CI - 180.9, - 81.2) among middle-educated women, and a $160 \mathrm{~g}$ lower birth weight among low-educated women (95\% CI -271.4, $-50 \cdot 2$; Fig. 3). Compared to non-smoking women with high adherence to the Mediterranean diet, smoking during pregnancy combined with high adherence was associated with a $66 \mathrm{~g}$ lower birth weight (95\% CI $-130 \cdot 6,-2 \cdot 5)$. In contrast, birth weight was approximately $214 \mathrm{~g}$ lower in women who continued to smoke during pregnancy with low adherence to the diet (95\% CI $-269 \cdot 3,-159 \cdot 6)$. No further significant effect of modification on the additive scale was observed for parity, BMI and folic acid use (all interaction terms $P>0 \cdot 10$ ).

\section{Discussion}

To our knowledge, this is one of the first studies to examine the relationship between dietary habits in early pregnancy and fetal and placental growth during intra-uterine life. Degree of adherence to a Mediterranean-like diet is significantly associated with intra-uterine growth parameters revealing a higher birth weight and placental weight for women with strong adherence to the diet.

The use of dietary pattern analysis has been commonly accepted as it provides essential and complementary insights into overall dietary behaviour. The major challenge remains to establish a quantitative method to identify eating patterns that are associated with disease risk. First, similar to the conventional principal component factor extraction technique logistic regression is an alternative method to extract directions, i.e. combinations of variables. However, in logistic regression analysis, the directions are selected based on their ability to predict a binary response variable ${ }^{(21)}$. Multicollinearity is not considered as a threat to the validity of the solution because here, only mild correlations were observed between the food groups (range of variance inflation factors 1.032.03). Second, as we only used the method to predict, it is highly unlikely that this will have caused a problem. Also, the results were cross-validated within a random subgroup 

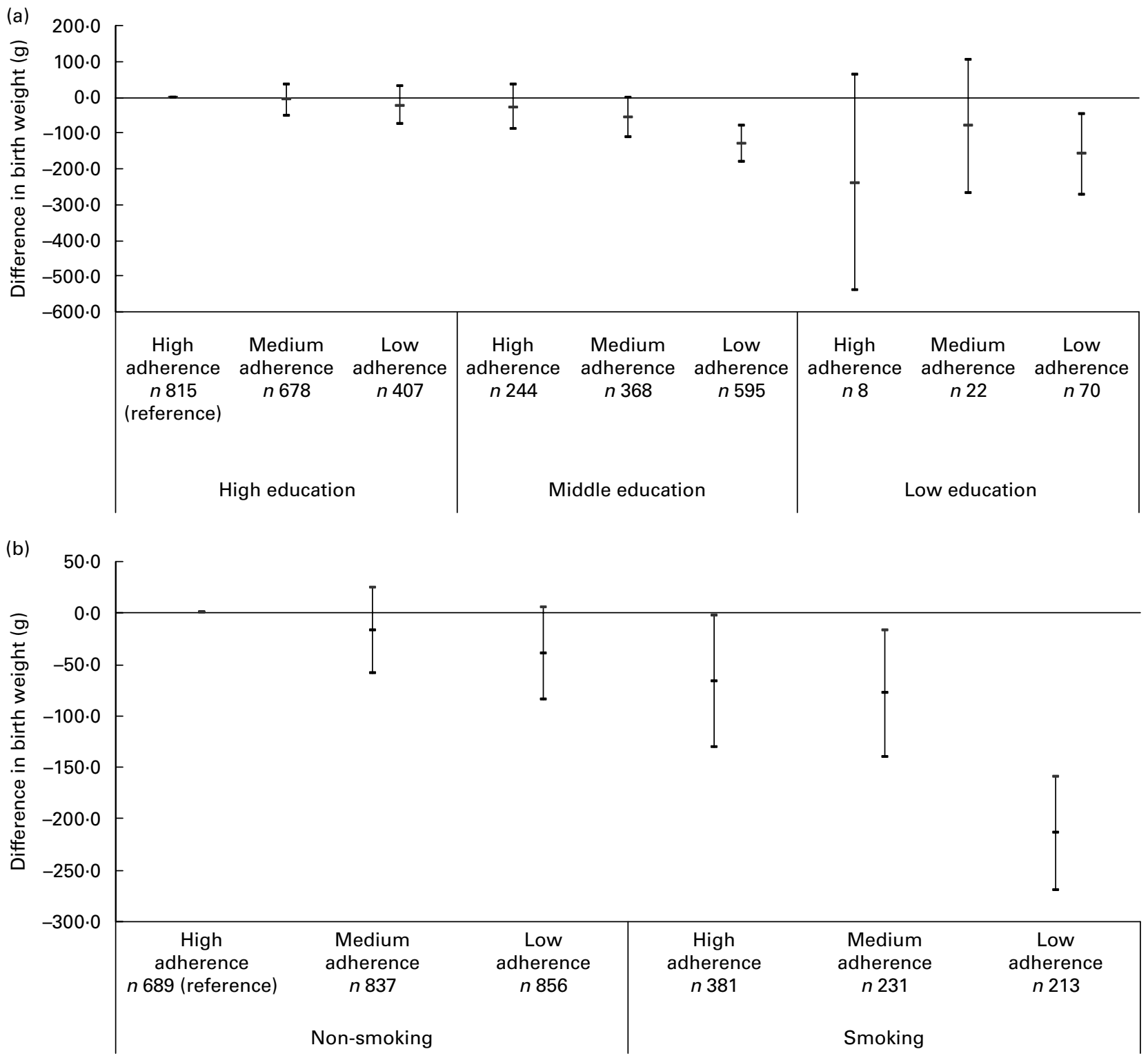

Fig. 3. Associations between use of the degree of adherence to the Mediterranean diet and birth weight, stratified by (a) education and (b) smoking. Results from linear regression analyses stratified per dietary adherence/educational level; dietary adherence/smoking habits. Values are regression coefficients (95\% $\mathrm{Cl}$ ) and reflect the difference in birth weight in grams, compared with reference. All values are adjusted for maternal age, height, weight, parity, sex, folic acid use, education (only analysis regarding smoking), and smoking (only analysis regarding education).

providing comparable solutions. Next, the addition or deletion of one or two food groups did not affect the general pattern of findings much. Nevertheless, we also performed a conventional principal component analysis that resulted in eight relevant factors of which the first factor, showing the best similarities with the dietary pattern identified with the logistic regression solution, was associated with IUGR with an OR of 0.829 (95\% CI 0.731, 0.941). The correlation between this first factor and the score from the logistic regression solution was $r-0.38$. (Table S1, supplementary material for this article can be found at http://www.journals.cambridge.org/bjn). As the outcome (IUGR) was dichotomous (yes/no (0/1)), reduced rank regression was not applied since this method is used for multiple continuous outcomes.
Nutritional studies are always prone to some bias, including imprecise measurement of nutritional intake. Several studies compared the results of dietary pattern analysis using FFQ with those using weighted dietary records, and observed no differences $^{(22)}$. Moreover, in prospective studies, with the exposure measured before occurrence of the outcome, imprecise measurement of nutritional intake is likely to be random. Another limitation is the potential for residual confounding. Although we attempted to account for this by restricting to an ethnic homogeneous population and by controlling for a large number of confounders, we cannot rule out that residual confounding occured ${ }^{(23)}$. Thus, our findings should be viewed with care. Lastly, nutritional intake was assessed in early pregnancy because of evidence that the trajectory of fetal growth 
and development is set at this stage ${ }^{(1,2)}$. It could be argued that nutritional intake differs throughout pregnancy. However, Cucó et $a l^{(24)}$ investigated dietary patterns during different pregnancy periods and observed no significant differences over time.

Over the past years, the Mediterranean diet has gained considerable attention for its positive health effects. There is no single Mediterranean diet. However, dietary patterns that prevail in the Mediterranean region share common characteristics including high intakes of vegetables and vegetables oil, moderate amounts of fish, poultry and alcohol, and relatively low consumptions of meat ${ }^{(25)}$. Adherence to the Mediterranean-like diet in our study was reflected by relatively high amounts of vegetable protein, carbohydrate polymers, fibre, and a favourable ratio of unsaturated:saturated lipids. Increasing concentrations of the biomarkers folate and vitamin $\mathrm{B}_{12}$, and decreasing tHcy concentrations further validated adherence to the diet ${ }^{(26)}$. These biomarker concentrations partly represent a mother's dietary intake as they also depend on lifestyle, genetic factors, and endocrine- and metabolic functions ${ }^{(23)}$.

The B-vitamins folate and vitamin $\mathrm{B}_{12}$ serve as substrates and cofactors in several pathways of cellular processes, including cell multiplication, apoptosis and intracellular signalling ${ }^{(27)}$. These processes can also be affected by saturated fats, trans-fatty acids and cholesterol ${ }^{(28)}$. For this reason, adherence to a dietary pattern characterised by relatively high concentrations of these B-vitamins and a favourable lipid profile is likely to influence intra-uterine growth. The results of this study are comparable with our previous findings on folic acid use in relation to infant low birth weight ${ }^{(29)}$. The results are further supported by another study showing that the use of a dietary pattern rich in vegetables, fruit, poultry and fish was associated with a $25 \%$ reduced risk of IUGR, as compared to a dietary pattern rich in meat, dairy products, snacks and potatoes ${ }^{(7)}$. It is noteworthy that this latter study primarily focused on birth weight as a proxy for fetal growth. Yet, birth weight is the end-point of different growth patterns determined by multiple constitutional and environmental factors. Studying fetal size trajectories may better distinguish growth-restricted from constitutionally small infants.

Both restricted and accelerated fetal growth have been linked with alterations in placental development and function $^{(30)}$. In the present study, women with low adherence to the Mediterranean diet did not only have smaller placentas but also tended towards higher uteroplacental vascular resistance. This seems biologically plausible since early placentation is characterised by vascular remodelling, increased inflammation, oxidative stress and rapid cell division ${ }^{(31,32)}$. In both pregnant and non-pregnant populations, these processes have been linked to nutrients associated with the Mediterranean dietary pattern, including folate, $n-3$ fatty acids and antioxidant vitamins ${ }^{(6,33)}$. Moreover, comparable dietary patterns have been demonstrated to reduce markers of inflammation and endothelial dysfunction, including C-reactive protein and E-selectin ${ }^{(6,33,34)}$. Low concentrations of antioxidant vitamins, $\mathrm{Mg}$ and fibre may underlie these biological processes.
The Mediterranean diet is an important source of methyl donors. Differences in quantitative methylation may affect genes implicated in placental and fetal size. Our results might also suggest that fetal and placental programming can be affected by use of the Mediterranean dietary pattern in early pregnancy $^{(2)}$. This is supported by recent findings that periconception folic acid use is associated with epigenetic changes in the insulin-like growth factor 2 gene in the child, thereby potentially affecting intra-uterine programming ${ }^{(35)}$. This could also apply to our results, suggesting that maternal diet may cause epigenetic modifications in the embryo, resulting in altered growth patterns. However, at this moment, these underlying mechanisms are just starting to be explored in humans.

Finally, the association between the degree of adherence to the Mediterranean diet and birth weight differed according to educational level. The relationship between socio-economic status and birth weight has been well established ${ }^{(23,36)}$. In general, low-educated women practise a less healthy lifestyle ${ }^{(37)}$. It is conceivable that poor dietary habits of low-educated women exacerbated the potential harmful effects of other unhealthy behaviours on fetal size. In this respect, we also reported on the modifying effect of smoking in this study sample. Previously, Jaddoe et al. ${ }^{(17)}$ showed that smoking during pregnancy impaired fetal size. Smoking during pregnancy has also been reported to induce morphological and functional changes in the placenta leading to a reduction in fetal-placental blood flow ${ }^{(38)}$. We recently observed that smoking modified the effect of folic acid use on first trimester growth $^{(39)}$. This could suggest a significant role for folate in our observed associations.

In conclusion, the degree of adherence to the Mediterranean diet is associated with several features of intra-uterine growth resulting in a lower placental weight and a lower birth weight for women with a low adherence. These results substantiate the importance of the Mediterranean diet and suggest the need for more attention and awareness in pregnancy. Further research is warranted to study the effects of dietary patterns and their interference on underlying epigenetic mechanisms and subsequent consequences for postnatal growth and future health.

\section{Acknowledgements}

The Generation R Study was conducted by the Erasmus Medical Center in close collaboration with the School of Law and Faculty of Social Sciences of the Erasmus University Rotterdam, the Municipal Health Service Rotterdam area, Rotterdam, the Rotterdam Homecare Foundation, Rotterdam and the Stichting Trombosedienst \& Artsenlaboratorium Rijnmond (STAR), Rotterdam. The authors gratefully acknowledge the contribution of general practitioners, hospitals, midwives and pharmacies in Rotterdam. The first phase of the Generation R Study was financed by the Erasmus Medical Center, Rotterdam, the Erasmus University Rotterdam and the Netherlands Organization for Health Research (ZonMw). Vincent Jaddoe also acknowledges the personal funding by ZonMw (ZonMw 90700303). The contributions of the authors to the present study were as follows: R. P. S.-T., J. M., A. H., E. E. L., 
V. V. J. and E. A. S. designed the research; S. T., R. P. S.-T., M. V., H. d. B., V. V. J. and E. A. S. conducted the research; H. R., J. L., J. M., A. H. and E. E. L. provided essential reagents, or essential materials; S. T., R. P. S.-T., M. V., H. d. B., H. R., J. L. and V. V. J. analysed the data, or performed statistical analysis; S. T., R. P. S.-T., M. V., V. V. J. and E. A. S. wrote the paper; E. A. S. had primary responsibility for the final content. All authors read and approved the final manuscript. The authors declare that there are no personal or financial conflicts of interest.

\section{References}

1. Gluckman PD, Hanson MA, Cooper C, et al. (2008) Effect of in utero and early-life conditions on adult health and disease. $N$ Engl J Med 359, 61-73.

2. Waterland RA \& Michels KB (2007) Epigenetic epidemiology of the developmental origins hypothesis. Annu Rev Nutr 27, 363-388.

3. Vujkovic M, Steegers EA, Looman CW, et al. (2009) The maternal Mediterranean dietary pattern is associated with a reduced risk of spina bifida in the offspring. BJOG 116, 408-415.

4. Haugen M, Meltzer HM, Brantsaeter AL, et al. (2008) Mediterranean-type diet and risk of preterm birth among women in the Norwegian Mother and Child Cohort Study (MoBa): a prospective cohort study. Acta Obstet Gynecol Scand 87, 319-324.

5. Mikkelsen TB, Osterdal ML, Knudsen VK, et al. (2008) Association between a Mediterranean-type diet and risk of preterm birth among Danish women: a prospective cohort study. Acta Obstet Gynecol Scand 87, 325-330.

6. Nettleton JA, Steffen LM, Mayer-Davis EJ, et al. (2006) Dietary patterns are associated with biochemical markers of inflammation and endothelial activation in the Multi-Ethnic Study of Atherosclerosis (MESA). Am J Clin Nutr 83, 369-379.

7. Knudsen VK, Orozova-Bekkevold IM, Mikkelsen TB, et al. (2008) Major dietary patterns in pregnancy and fetal growth. Eur J Clin Nutr 62, 463-470.

8. Jaddoe VW, Bakker R, van Duijn CM, et al. (2007) The Generation R Study Biobank: a resource for epidemiological studies in children and their parents. Eur J Epidemiol 22, 917-923.

9. Jaddoe VW, van Duijn CM, van der Heijden AJ, et al. (2008) The Generation R Study: design and cohort update until the age of 4 years. Eur J Epidemiol 23, 801-811.

10. The International Response to Helsinki VI (2000) The WMA's Declaration of Helsinki on Ethical Principles for Medical Research Involving Human Subjects, as adopted by the 52nd WMA General Assembly, Edinburgh.

11. Klipstein-Grobusch K, den Breeijen JH, Goldbohm RA, et al. (1998) Dietary assessment in the elderly: validation of a semiquantitative food frequency questionnaire. Eur J Clin Nutr 52, 588-596.

12. Donders-Engelen M, Heijden van der L \& Hulshof KF (2003) Maten, Gewichten en Codenummers. Wageningen: Human Nutrition of TNO and Wageningen University.

13. Netherlands Nutrition Center (2006) Nevo: Dutch Food Composition Database 2006. The Hague: Netherlands Nutrition Center.

14. Verburg BO, Steegers EA, De Ridder M, et al. (2008) New charts for ultrasound dating of pregnancy and assessment of fetal growth: longitudinal data from a populationbased cohort study. Ultrasound Obstet Gynecol 31, 388-396.
15. Verburg BO, Mulder PG, Hofman A, et al. (2008) Intra- and interobserver reproducibility study of early fetal growth parameters. Prenat Diagn 28, 323-331.

16. Statistics Netherlands (2004) Standaard Onderwijsindeling 2003 (Standard Classification of Education 2003). Voorburg/Heerlen

17. Jaddoe VW, Verburg BO, de Ridder MA, et al. (2007) Maternal smoking and fetal growth characteristics in different periods of pregnancy: the generation R study. $\mathrm{Am}$ J Epidemiol 165, 1207-1215.

18. Willett W \& Stampfer MJ (1986) Total energy intake: implications for epidemiologic analyses. Am J Epidemiol 124, $17-27$.

19. Sterne JA, White IR, Carlin JB, et al. (2009) Multiple imputation for missing data in epidemiological and clinical research: potential and pitfalls. BMJ 338, 2393.

20. Rubin DB (1987) Multiple Imputation for Nonresponse Data in Surveys. New York, NY: John Wiley \& Sons, Inc.

21. Roy S, Lavine J, Chiaromonte F, et al. (2009) Multivariate statistical analyses demonstrate unique host immune responses to single and dual lentiviral infection. PLoS One 4, 7359.

22. Hu FB, Rimm E, Smith-Warner SA, et al. (1999) Reproducibility and validity of dietary patterns assessed with a foodfrequency questionnaire. Am J Clin Nutr 69, 243-249.

23. Park SY, Murphy SP, Wilkens LR, et al. (2005) Dietary patterns using the Food Guide Pyramid groups are associated with sociodemographic and lifestyle factors: the multiethnic cohort study. J Nutr 135, 843-849.

24. Cuco G, Fernandez-Ballart J, Sala J, et al. (2006) Dietary patterns and associated lifestyles in preconception, pregnancy and postpartum. Eur J Clin Nutr 60, 364-371.

25. Balder HF, Virtanen M, Brants HA, et al. (2003) Common and country-specific dietary patterns in four European cohort studies. J Nutr 133, 4246-4251.

26. Gao X, Yao M, McCrory MA, et al. (2006) Dietary pattern is associated with homocysteine and B vitamin status in an urban Chinese population. J Nutr 133, 636-642.

27. Tamura $\mathrm{T} \&$ Picciano MF (2006) Folate and human reproduction. Am J Clin Nutr 83, 993-1016.

28. Larque E, Zamora S \& Gil A (2001) Dietary trans fatty acids in early life: a review. Early Hum Dev 65, S31-S41.

29. Timmermans S, Jaddoe VW, Hofman A, et al. (2009) Periconception folic acid supplementation, fetal growth and the risks of low birth weight and preterm birth: the Generation R Study. Br J Nutr 102, 777-785.

30. Bleker OP, Buimer M, van der Post JA, et al. (2006) Kloosterman: on intrauterine growth. The significance of prenatal care. Studies on birth weight, placental weight and placental index. Placenta 27, 1052-1054.

31. Goodger AM \& Rogers PA (1993) Uterine endothelial cell proliferation before and after embryo implantation in rats. J Reprod Fertil 99, 451-457.

32. Burton GJ \& Jaunaiux E (2001) Maternal vascularisation of the human placenta: does the embryo develop in a hypoxic environment? Gynecol Obstet Fertil 29, 503-508.

33. Esposito K, Marfella R, Ciotola M, et al. (2004) Effect of a mediterranean-style diet on endothelial dysfunction and markers of vascular inflammation in the metabolic syndrome: a randomized trial. JAMA 292, 1440-1446.

34. Du H, van der AD, van Bakel MM, et al. (2008) Glycemic index and glycemic load in relation to food and nutrient intake and metabolic risk factors in a Dutch population. Am J Clin Nutr 87, 655-661.

35. Steeegers-Theunissen RPM, Obermann-Borst SA, Kremer D, et al. (2009) Periconceptional maternal folic acid use of 400 micrograms per day is related to increased methylation 
of the IGF2 gene in the very young child. PLoS One 4, 7845 $1-78455$.

36. Jansen PW, Tiemeier H, Looman CW, et al. (2009) Explaining educational inequalities in birthweight: the Generation $\mathrm{R}$ Study. Paediatr Perinat Epidemiol 23, 216-228.

37. Laaksonen M, Talala K, Martelin T, et al. (2008) Health behaviours as explanations for educational level differences in cardiovascular and all-cause mortality: a follow-up of
60000 men and women over 23 years. Eur J Public Health 18, 38-43.

38. Zdravkovic T, Genbacev O, McMaster MT, et al. (2005) The adverse effects of maternal smoking on the human placenta: a review. Placenta 26, S81-S86.

39. Mook-Kanamori DO, Steegers EA, Eilers PH, et al. (2010) Risk factors and outcomes associated with first-trimester fetal growth restriction. JAMA 303, 527-534. 